\title{
Traduire
}

Revue française de la traduction

$214 \mid 2007$

L'art de l'invisible

\section{La traduction à Interpol}

\section{Muriel Millet}

\section{OpenEdition}

\section{Journals}

Édition électronique

URL : http://journals.openedition.org/traduire/1382

DOI : 10.4000/traduire.1382

ISSN : 2272-9992

\section{Éditeur}

Société française des traducteurs

\section{Édition imprimée}

Date de publication : 1 juin 2007

Pagination : 5-14

ISSN : 0395-773X

\section{Référence électronique}

Muriel Millet, « La traduction à Interpol », Traduire [En ligne], 214 | 2007, mis en ligne le 01 juin 2007, consulté le 21 avril 2019. URL : http://journals.openedition.org/traduire/1382 ; DOI : 10.4000/ traduire.1382 


\title{
La traduction à Interpol
}

\author{
MuRiel Millet \\ Chef du département linguistique français, OIPC, Interpol
}

Vaste sujet que celui de la pratique de la traduction dans une organisation internationale, dont l'enjeu, s'agissant d'Interpol, est de favoriser la communication entre 186 pays membres. Afin d'appréhender tous les tenants et les aboutissants de cette activité, il convient de présenter brièvement l'organisation au sein de laquelle elle s'exerce. Les services linguistiques d'Interpol feront l'objet de la deuxième partie, tandis que la troisième partie sera consacrée à la problématique de la traduction telle qu'elle se pratique dans l'Organisation.

\section{Quelques mots sur Interpol}

Interpol a été créé en 1923 et est, avec 186 pays membres, la plus grande organisation de police au monde et la deuxième organisation internationale après les Nations Unies si l'on considère le nombre de pays qui en sont membres. Ses buts sont :

a) D'assurer et de développer l'assistance réciproque la plus large de toutes les autorités de police criminelle, dans le cadre des lois existant dans les différents pays et dans l'esprit de la Déclaration universelle des droits de l'homme ;

b) D'établir et de développer toutes les institutions capables de contribuer efficacement à la prévention et à la répression des infractions de droit commun.

De plus, l'article 3 de son Statut lui interdit toute activité ou intervention dans des questions ou affaires présentant un caractère 
politique, militaire, religieux ou racial. Sa mission est de faciliter la coopération transfrontalière entre les services de police, et d'apporter appui et assistance à tous les services, organisations et autorités ayant pour mission de prévenir et de combattre la criminalité, et ce, dans le respect des six principes régissant la coopération.

Interpol a cinq domaines de criminalité prioritaires : les stupéfiants et la criminalité organisée, la criminalité financière liée aux technologies de pointe, la recherche des malfaiteurs en fuite, la sûreté publique et le terrorisme, et le trafic d'êtres humains. D'autres domaines tels que la corruption, les atteintes à la propriété intellectuelle et à l'environnement et les infractions contre les biens font aussi l'objet d'un intérêt particulier.

Le Secrétariat général se trouve à Lyon. Interpol compte six bureaux régionaux - Argentine, Côte d'Ivoire, El Salvador, Kenya, Thaillande et Zimbabwe - ainsi qu'une représentation aux Nations Unies. Chaque pays membre a un Bureau central national (BCN) dont l'effectif se compose de policiers qui dépendent de leur administration nationale. Le BCN est l'interlocuteur désigné du Secrétariat général, des bureaux régionaux et des autres pays membres qui sollicitent une assistance pour leurs enquêtes à l'étranger ainsi que pour la localisation et l'arrestation de malfaiteurs en fuite.

Le Règlement général de l'Organisation prévoit, dans son article 54, que ses langues de travail sont l'anglais, l'arabe, l'espagnol et le français.

\section{Les services linguistiques à Interpol}

Dans l'organigramme, ils dépendent de la direction de l'Administration et du Budget. Au total, 36 personnes y travaillent, soit environ $7 \%$ de l'effectif de l'Organisation. Il faut dire que la charge de travail a connu une forte augmentation entre 2000 et 2005 (derniers chiffres disponibles), augmentation qui s'est traduite par un recours accru aux traducteurs extérieurs et à du personnel temporaire. Nos normes de 
performance se situent entre cinq et six pages de 300 mots par jour, suivant la difficulté des documents. Notre démarche est avant tout axée sur la qualité (plus que sur les statistiques) mais tant la nature des travaux que nos méthodes et outils de travail ont fortement évolué ces dernières années.

\section{Nature des travaux de traduction effectués à Interpol}

Le gros de la charge de travail des services linguistiques est constitué par ce que l'on appelle les notices, qui en représentent $60 \%$. Les notices servent à échanger des informations de police essentielles concernant des individus recherchés pour des infractions graves, des personnes disparues, des cadavres non identifiés, d'éventuelles menaces ainsi que les modus operandi employés par les malfaiteurs. Il en existe sept sortes différentes et la plus connue d'entre elles, celle dont on entend parler dans les médias, est la notice rouge, qui est utilisée pour demander l'arrestation ou l'arrestation provisoire en vue d'extradition d'individus recherchés. Les notices contiennent des informations de deux types :

- Des éléments d'identification : état civil, signalement, photographie, empreintes et autres informations utiles telles que profession, langues parlées, numéros des pièces d'identité, etc. ;

- Des éléments juridiques : qualification de l'infraction dont est accusé l'individu concerné, références des dispositions de la loi pénale réprimant l'infraction, peine infligée ou peine maximale encourue, etc.

Les services linguistiques d'Interpol traduisent également tous les documents préparatoires aux réunions des organes de l'Organisation : Assemblée générale, Comité exécutif, conférences régionales. Ces documents traitent d'une grande diversité de sujets : financiers (budget et rapport financier d'Interpol, par exemple), juridiques (défense des intérêts de l'Organisation, accords de coopération, règlement de différends entre pays, etc.), techniques (développement de nouveaux systèmes d'information et de communication, interfaces de bases de données, police scientifique), politiques (coopération inter- 
nationale), etc. À cela s'ajoutent les publications de l'Organisation : guides et manuels de toutes sortes (Manuel Interpol de bonne pratique à l'usage des policiers spécialisés dans les enquêtes sur la pédocriminalité, Guide O.I.P.C.-Interpol de l'analyse criminelle, Guide Interpol sur l'échange de données génétiques et sur les pratiques en matière d'analyse d'ADN, etc.), comptes rendus de réunions et de conférences, rapports d'analyse sur des opérations de police ou des phénomènes de criminalité particuliers, articles sur des sujets spécialisés. Enfin, on ne peut passer sous silence tous les documents dits "de communication ", consultables sur le site de l'Organisation, tels que les communiqués de presse, les fiches pratiques et juridiques, les brochures et plaquettes de présentation, etc.

\section{Les outils à la disposition des traducteurs à Interpol}

C'est dire la somme de connaissances que doit posséder le traducteur s'il veut effectuer un travail de qualité. En effet, il ne s'agit pas seulement de rendre dans une langue $B$ (la langue cible) un mot ou une phrase exprimés dans une langue A (la langue source). Il faut faire en sorte que la traduction donne l'impression d'être un texte original, produit par un spécialiste de la question traitée. En d'autres termes, le traducteur doit produire, dans une langue d'arrivée, un texte ayant les mêmes fonctionnalités que le texte dans la langue de départ. Pour traduire convenablement un texte, il faut donc le comprendre, et pour le comprendre, il faut bien souvent faire des recherches terminologiques et documentaires. C'est pourquoi le traducteur dispose d'une panoplie d'outils dont il va chercher à tirer le meilleur parti :

- Les dictionnaires - qu'il s'agisse des dictionnaires généralistes, mono ou bilingues, ou des dictionnaires spécialisés, ces ouvrages restent incontournables, véritables "béquilles" toujours disponibles même en cas de panne de réseau informatique!

- Internet, ses moteurs de recherche et ses sites spécialisés qui permettent un accès immédiat à l'information. 
- Les bases de données terminologiques - soit celles des autres organisations accessibles par l'intermédiaire du site JIAMCATT ${ }^{(1)}$, dont Interpol est membre, soit les glossaires élaborés en interne.

- Les outils informatiques d'aide à la traduction : à Interpol, nous utilisons MultiTrans, un système de reconnaissance de texte qui se caractérise par un corpus multilingue plein texte intégré à une infrastructure de gestion terminologique : ce système permet de créer des recueils de documents de référence (les textes de départ et les textes d'arrivée correspondants) et d'y accéder ensuite. L'alignement et l'indexation plein texte se font automatiquement, de même que l'extraction des expressions répétitives. Une fonction de recherche permet de retrouver toutes les occurrences d'une expression voulue, de les consulter dans leur contexte initial et d'en afficher les traductions préalables. Une autre fonction de ce système donne accès à des outils d'extraction et de création terminologique à partir de corpus existants ou d'information terminologique importée de diverses sources. Nous disposons également d'un logiciel de comparaison de textes, DeltaView, qui est très utile lorsque des modifications sont apportées sur une version préexistante d'un texte et que ces modifications ne sont pas (toutes) signalées.

- Les services d'un terminologue spécialisé.

- La possibilité de consulter les spécialistes et les rédacteurs des textes à traduire en cas d'ambiguïté. Ainsi, dans le département linguistique français, la consultation de policiers francophones permet souvent de valider un choix terminologique. On le sait, un concept (un signifié) devrait être désigné par un seul et même terme (le signifiant) tout au long du document à traduire. Consulter un spécialiste

(1) La plate-forme JIAMCATT (Joint Inter-Agency Meeting on Computer-Assisted Translation and Terminology), créée en 1987 à l'Office des Nations Unies à Genève, permet aux services linguistiques des différentes organisations internationales d'échanger des informations utiles à la traduction. Pour plus d'informations, consulter le site Internet : http://jiamcatt.unsystem.org/english/jiamcate.htm, NdIR. 
permet donc d'effectuer le choix du " meilleur " terme ou à tout le moins, de faire en sorte qu'il participe à la validation finale du terme en question.

- La mise au point de macros et le prétraitement de certains textes par le service de traitement des documents. Le prétraitement s'applique aux notices, évoquées plus haut, qui comportent des rubriques standard se répétant d'une notice à l'autre. Il consiste en la substitution de parties de phrases, de termes et de phrases entières à l'aide d'une macro mise au point en interne et permet d'importants gains de productivité eu égard au volume de travail que représente ce type de document.

- La formation - en donnant aux traducteurs la possibilité de se former, en interne ou en suivant des cours à l'extérieur de l'Organisation (à l'ETI de Genève par exemple), Interpol contribue au maintien de la qualité des documents multilingues.

- Les contacts avec les services linguistiques d'autres organisations, par exemple par l'intermédiaire de JIAMCATT, permettent une meilleure harmonisation et contribuent à la diffusion des connaissances en matière d'outils d'aide à la traduction.

L'objectif de ces outils est de permettre un accès rapide et convivial à l'information, et de traduire uniquement ce qui n'a jamais été traduit. Cette approche procède donc d'une recherche de l'efficacité et présente des avantages divers et variés : plus grande cohérence, mise en commun des ressources, élimination des redondances terminologiques et linguistiques, diminution des temps de recherche, économies d'échelle, répartition du travail selon les spécialisations de chacun.

\section{Problématique de la traduction à Interpol}

\section{L'antagonisme entre rapidité et qualité.}

À Interpol, nous sommes bien souvent obligés de travailler dans l'urgence. Nous sommes directement en prise sur l'actualité et nous 
devons nous montrer très réactifs. Les communiqués de presse deviennent très rapidement obsolètes ; les projets de décisions et de résolutions d'assemblées délibérantes doivent être traduits immédiatement car la traduction constitue une étape incontournable du processus décisionnel. En d'autres termes, sans elle, tous les pays d'une organisation internationale ne peuvent participer à la prise de décisions. Or, la traduction nécessite de la réflexion, des recherches, des vérifications pour produire un travail de qualité. Lorsque les documents doivent être produits dans l'urgence, le risque d'erreurs est important, d'autant plus qu'il n'est pas toujours possible de recourir à la révision.

\section{Laugmentation du nombre de documents traités en autorévision}

Pour les raisons exposées ci-avant, devant la forte augmentation de notre charge de travail, nous avons mis en place une politique en matière de révision, en vertu de laquelle font l'objet d'une révision après traduction :

a) Les textes qui engagent la responsabilité juridique, financière, politique et médiatique de l'Organisation ;

b) Les recommandations, résolutions, etc. des organes délibérants de l'Organisation et des groupes consultatifs ou d'experts créés par ceux-ci ;

c) Les documents traduits par un traducteur débutant à Interpol ;

d) À la demande du traducteur, tout document complexe présentant des difficultés particulières, notamment techniques, ou contenant des informations "sensibles";

e) Les documents longs partagés entre plusieurs traducteurs, à des fins d'harmonisation ;

f) Les documents faisant l'objet de nombreuses modifications en cours de traduction, afin de s'assurer que toutes les modifications ont été prises en compte.

Sont traités en autorévision :

a) Les textes courts ou répétitifs ne présentant pas de difficultés particulières; 
b) Les adaptations ou mises à jour de documents existants ;

c) Les textes traduits par un réviseur ou par un traducteur ayant une excellente connaissance du sujet traité et qui juge que son travail n'a pas besoin d'une révision.

Pour ce qui est des documents traduits par des prestataires extérieurs, ils font en principe l'objet d'un contrôle en interne afin de s'assurer qu'ils sont conformes aux usages et à la terminologie en vigueur à Interpol. Cependant, le recours à la sous-traitance implique que le document ne peut être traité en interne pour des raisons de temps, et il arrive fréquemment que les délais imposés ne permettent pas qu'il fasse l'objet des contrôles nécessaires dans le département linguistique concerné. Dans tous les cas, les documents traités à l'extérieur portent une mention indiquant qu'ils n’ont été ni traduits ni révisés par les services linguistiques de l'Organisation. Il est à noter qu'il est souvent dérogé aux principes énoncés ci-dessus, notamment en cas d'urgence. De plus, les départements linguistiques étant de petites structures, les réviseurs et les chefs de département effectuent souvent eux-mêmes des travaux de traduction, en particulier de textes complexes ou importants. L'organisation du travail vise avant tout à faire preuve d'adaptabilité en vue de produire un travail de qualité dans les délais requis.

\section{L'harmonisation et la normalisation de la terminologie}

La traduction de textes spécialisés (techniques, scientifiques, économiques, etc.) nécessite rigueur et précision. La terminologie employée ne doit laisser de place à aucun doute ni à aucune ambiguïté, d'où la nécessité d'une normalisation et d'une harmonisation. La normalisation en matière de terminologie revêt deux aspects : d'une part, l'établissement des formes terminologiques les plus adaptées pour représenter les concepts (normalisation formelle) et, d'autre part, la fixation des notions et des relations qui existent entre elles dans un domaine de spécialité déterminé (normalisation conceptuelle). Dans ce domaine, les traducteurs doivent faire face à des difficultés diverses : 
l'absence d'organisations ou de structures s'occupant de normalisation formelle et le manque de formation des spécialistes dans le domaine de la terminologie (lesquels connaissent le concept mais ne savent pas le nommer). Trop souvent, le traducteur perd du temps lorsqu'il s'agit de rechercher de la terminologie et de la documentation, et une meilleure harmonisation permettrait de résoudre ce problème. Or, s'il peut aujourd'hui puiser dans des mémoires de traduction (grâce à MultiTrans) et garantir ainsi l'homogénéité phraséologique et terminologique des textes produits par l'Organisation, synonyme de gains de qualité et de productivité accrue, il doit faire face au problème des occurrences multiples, particulièrement lorsque les textes qui alimentent le système sont traités en autorévision et que le temps manque pour réaliser les opérations de " toilettage » qui s’imposent.

\section{L'inégalité de traitement entre les langues et une tendance à favoriser le monolinguisme}

Malheureusement, les ressources financières et humaines qui permettraient d'accorder un traitement égal à toutes les langues de travail d'Interpol ne sont pas disponibles. Alors qu'elles sont énoncées clairement dans le Statut de l'Organisation, les langues de travail de celle-ci ne bénéficient pas du même traitement. La prépondérance de l'anglais est manifeste, comme en témoigne par exemple le site www.interpol.int, qui n'existe qu'en une seule langue mais où l'on trouve quand même quelques documents dans les quatre langues. Ainsi, nombre de rédacteurs dont la langue maternelle est l'une des langues de travail de l'Organisation rédigent leurs documents en anglais alors qu'ils ne le maitrisent pas forcément. Il en résulte une perte de qualité, privant Interpol d'informations et de connaissances souvent essentielles, et des imprécisions manifestes dont les auteurs ne prennent conscience qu'à la lecture de la version de leur rapport traduite dans leur langue, dont ils attribuent la pauvreté langagière au traducteur! D'une manière générale, la rédaction des documents par des auteurs qui ne maittrisent pas la principale langue de travail de l'Organisation est susceptible d'être source d'erreurs de traduction. 


\section{Conclusion}

Le métier de traducteur est souvent méconnu et mal compris. Pourtant, à Interpol comme dans les autres organisations internationales, et malgré la tendance au monolinguisme relevée ci-dessus, la traduction continue de jouer un rôle fondamental dans le processus décisionnel. En témoigne l'augmentation faramineuse de notre charge de travail ces dernières années. Notre métier a évolué puisque nous avons désormais accès quasi instantanément à l'information dont nous avons besoin. Les outils dont nous disposons aujourd'hui ont permis une forte augmentation de la productivité. Mais ne nous y trompons pas. La traduction en tant qu'outil de communication a encore de beaux jours devant elle car aucun système ne pourra jamais remplacer la force et la magie des mots.

\section{Sources}

www.interpol.int

Politique en matière de révision adoptée par les services linguistiques de l'Organisation en 2002.

Colomer Artigas Rosa et Bover Salvado Jordi, La normalización conceptual de los términos especializados: problemas y soluciones, Conférence sur la coopération dans le domaine de la terminologie en Europe, Paris, 1999.

De Saint Robert Marie Josée, Aperçu de la place des langues néo-latines dans la communication spécialisée des organisations internationales, Congrès international sur les langues néo-latines dans la communication spécialisée, Mexique, 2002.

Muriel Millet est chef du Département linguistique français d'Interpol depuis 1993. Traductrice d'anglais et espagnol en français, ses missions consistent à planifier, organiser et superviser les travaux de traduction et révision du Département, contrôler la quantité de travail fourni et veiller au maintien des normes de qualité et de productivité. Elle est aussi en charge de la formation des traducteurs et réviseurs du Département et dirige les recherches en matière de terminologie. 